\section{Schweizerisches Energieforschungsprogramm Elektrochemie}

\section{Otto Haas*}

Abstract. The need of advanced power sources especially for electric vehicle and load leveling applications motivated the Swiss government to promote a research program in electrochemistry. This program concentrates on research of electrode and electrolyte materials which are of interest for the development of new energy-storage and conversion devices such as advanced batteries, fuel cells, and electrolysers. The program involves researchers from different disciplines and institutes. Material research, electrochemical engineering, and system modeling are the main topics of the program. The material research is concentrating on electrode materials with high energy content like $\mathrm{Li}, \mathrm{Al}, \mathrm{Mg}$, $\mathrm{Ca}$, and insertion electrodes. Important research topics are also conducting polymers, redox polymers, and polyelectrolytes or solid electrolytes. In a later stage, the research achievements should be used to develop high power density batteries and fuel cells.

\section{Einleitung}

Gestützt auf sein Konzept der Energieforschung hat der Bund zusätzliche Forschungsmittel zur Verfügung gestellt. Verwaltet wird diese Forschung gemeinsam vom Bundesamt für Energiewirtschaft $(B E W)$ und dem Bundesamt für Bildung und Wissenschaft $(B B W)$.

Das Gebiet der chemischen Energiespeicherung wird von Dr. P. Kesselring (PSI) koordiniert und wurde in folgende Programme aufgeteilt: Thermochemie (Programmleiter: Dr. $A$. Reller, Universität Zürich), Photochemie (Dr. J.C. Courvoisier, 1225 Chêne-Bourg), Wasserstoff (PD Dr. Th. H. Schucan, Paul Scherrer Institut, Villigen), Elektrochemie (Dr. O. Haas, Paul Scherrer Institut, Villigen).

Das Programm Elektrochemie umfasst ein relativ grosses interdisziplinäres Gebiet mit Forschungsthemen, die vor allem im Zusammenhang mit der elektrochemischen Energie-Umwandlung und -Speicherung von Interesse sind.

Die Forschungsaktivität auf diesem Gebiet wurde in den letzten Jahren speziell in Japan, den Oststaaten und den USA stark forciert. Die Entwicklung neuer Stromquellen und Energie-Umwandlungssysteme wurde vor allem für die Raumfahrt und für militärische Zwecke vorangetrieben. Die in der Schweiz tätigen Batteriefabrikanten produzieren mit Ausnahme der Knopfbatterien praktisch nur konventionelle Batterien, wobei der harte internationale Konkurrenzkampf diesen Firmen wenig finanziellen Spielraum für Forschung und kostspielige Neuentwicklungen zulässt.

Für die sich abzeichnenden zivilen Anwendungen neuer Batterie- und Brenn- stoffzellensysteme sind Forschungs- und Entwicklungsanstrengungen nötig, die die Möglichkeiten unserer einheimischen Batterieindustrie bei weitem übersteigen.

Bei der Forschung auf dem Gebiet der elektrochemischen Energiespeicherung ergibt sich eine Reihe wissenschaftlich hochinteressanter Fragestellungen, die an Hochschulen und staatlichen Forschungsinstituten kompetent bearbeitet werden können. Die in den letzten Jahren erzielten Fortschritte in Materialtechnologie und 'Chemical Engineering' auf molekularem Niveau geben $\mathrm{zu}$ berechtigten Hoffnungen Anlass, dass neue Batterie-Systeme mit hohen Energie- und Leistungsdichten in absehbarer Zeit entwickelt werden können.

Durch die finanzielle Unterstützung des Bundes besteht nun die einmalige Chance, ein hochqualifiziertes, interdisziplinäres Forschungsteam zusammenzubringen, welches wichtige Beiträge zur Entwicklung neuer Systeme liefern kann. Dabei wird es im Moment weniger darum gehen, an einem bestehenden oder international in Entwicklung stehenden System Entwicklungsbeiträge zu leisten. Es sollten vielmehr neue, potentiell interessante Elektrodenmaterialien, Elektrolyte und Membranen entwickelt und an diesen Materialien grundlegende Studien durchgeführt werden. Von diesen Materialien sollten die für die Elektrochemie wichtigen Parameter gemessen und gezielt auf ihre potentielle Anwendung hin optimiert werden. In einer ersten Forschungsphase wird es wichtig sein, dass das nötige 'Werkzeug' zur Untersuchung von relevanten Vorgängen in elektrochemischen Zellen bereitgestellt wird. Dazu gehört einerseits die Entwicklung neuer in-situ-Analysenmethoden, mit denen die elektrochemischen Prozesse ver- folgt werden können, andererseits die Entwicklung numerischer Simulationsmethoden, die eine Modellierung der elektrochemischen Prozesse erlauben.

Erst in einer zweiten Phase sollten zusammenpassende Einzelkomponenten mit speziell guten Eigenschaften in ganzen Zellen getestet werden. Bei positivem Ergebnis könnten dann die eigentlichen BatterieEntwicklungsarbeiten beginnen. In diesem Stadium müsste sich die Industrie mitbeteiligen, da sonst weitere Investitionen fragwürdig werden.

Durch dieses Vorgehen, welches der Grundlagenforschung cinen grossen Spielraum lässt, können neue bahnbrechende Materialien gefunden werden, welche ebenfalls anderen Forschungsbereichen neue Impulse geben können. Die auf diesem Gebiet möglichen 'Spin-offs' für Sensoren, elektronische Anzeigen, Membrantechnologie, Informationsspeicherung usw. sind beträchtlich und rechtfertigen erhebliche Investitionen.

\section{Zielsetzung}

Das Programm 'Elektrochemie' soll die Zusammenarbeit von Forschern aus verschiedenen Disziplinen und Instituten fördern. Damit sollen Grundlagen für die Entwicklung neuer und die Verbesserung bestehender elektrochemischer Energiespeichersysteme geschaffen werden. Es sollen besonders Arbeiten unterstützt werden. die im Zusammenhang mit der Entwicklung von wiederaufladharen Batterien. Brennstoffzellen und Elektroly'seapparaturen zur Herstellung energiereicher Produkte stehen. Als Fernziel stehen die Anwendungen: Elektroauto, Spitzenstromausgleich, elektrochemische Energicumivandlung.

Die vorgesehenen Forschungsarbeiten und speziell die Entwicklungsarbeiten an fortgeschrittenen Systemen sollen wenn immer möglich in enger Zusammenarbeit mit der Industrie durchgeführt und gefördert werden.

\section{Forschungsrichtungen}

Das Forschungsprogramm kann grob in drei Forschungsrichtungen $(3.1-3.3)$ aufgeteilt werden:

\subsection{Untersuchung neuer Systeme}

Neue wiederaufladbare elektrochemische Zellen, $\mathrm{H}_{2} /$ Luft-Brennstoffzellen, Methanol/Luft-Brennstoffzellen, Metall/LuftZellen, thermisch regenerierbare Zellen.

\subsection{Materialforschung}

Untersuchung unkonventioneller Elektrolytsysteme, Festelektrolyte, Polymerelektrolyte, Schmelzen, aprotische orga-

* Korrespondenz: Dr. O. Haas

Paul-Scherrer-lnstitut

$\mathrm{CH}-5232$ Villigen PSI 
nische Elektrolyte, neue Elektrodenstrukturen, neue Zellfabrikationstechniken, neue Separatoren, ionenleitende Membranen, Gasdiffusionsmembranen, leitende Polymere, Redoxpolymere, Intercalationsund Insertionselektroden, Korrosionsverhalten der Zellmaterialien.

\subsection{Grundlagen für 'Electrochemical Engi- neering'}

Thermochemie und Thermodynamik der Zellreaktionen, Wärmehaushalt von Zellaggregaten, Massentransportphänomene, Elektrodenkinetik, Mechanismus des Elektrontransfer-Prozesses, DiffusionsKonvektions- und Migrationsprozesse, Ionentransport, Oberflächenmodifikation, Einfluss der Oberflächenstruktur auf die Elektrodenprozesse, numerische Simulation der Elektrodenprozesse, Modellierung von Elektrodenreaktionen und von ganzen elektrochemischen Zellen.

\section{Forschungsschwerpunkte}

Damit die einzelnen Forschungsgruppen voneinander profitieren können, sollten Forschungsschwerpunkte mit stark überlappenden Forschungsthemen geschaffen werden. Ein möglicher Forschungsschwerpunkt besteht zum Beispiel aus dem Themenkreis: leitende Polymere, Redoxpolymere, Membranen, Polyelektrolyte. Diese für die elektrochemische Energie-Umwandlung und -Speicherung wichtigen Materialien werden ähnlich hergestellt, haben verwandte Eigenschaften und können mit den gleichen Untersuchungsmethoden charakterisiert werden, obwohl sie in einer elektrochemischen Zelle stark verschiedene Funktionen zu erfüllen haben. Es ist deshalb sicher sinnvoll, wenn diese Punkte als Schwerpunktsthema ins Programm aufgenommen werden können und sich eine 'überkritische' Forschergruppe mit der Problematik dieser Materialien auseinandersetzt. Dabei sollten physikalische Grössen wie elektrische Leitfähigkeit, Ionentransport, Lösungsmitteltransport, elektrochemische Potentiale, Donnanpotentiale sowie Redoxzentrendichte und Massendichte gemessen werden. Zur Abschätzung der Brauchbarkeit dieser Materialien sind aber auch Kenntnisse der chemischen Eigenschaften wie die chemische Stabilität oder Daten über die Thermodynamik und Kinetik der Protonierungsgleichgewichte, Gegenionen- und Komplexierungsgleichgewichte nötig. Es sollte auch abgeklärt werden, wie weit diese physikalisch-chemischen Eigenschaften durch gezielte Strukturänderungen auf molekularem oder makromolekularem $\mathrm{Ni}$ veau beeinflusst werden können.

Stark überlappende Forschungsaktivitäten ergeben sich auch im Themenkreis: elektrochemische Untersuchung von Anodenmaterialien mit hoher Energiedichte, z. B. Li, Na, K, Mg, Ca, Al, Si usw., Herstellung energiereicher Produkte wie $\mathrm{H}_{2}$, $\mathrm{Al}, \mathrm{Mg}, \mathrm{Si}$. Für diese Arbeiten müssen ähn- liche Arbeitstechniken, wie z. B. Arbeiten mit Drybox und Umgang mit wasserfreien Lösungsmitteln zur Verhinderung einer kompakten, passivierenden Oberflächenoxid-Schicht, eingesetzt werden. Erfahrungen mit diesen Elektrodenmaterialien lassen sich oft von einem Material auf das andere übertragen. Forscher in diesem Themenkreis sollten deshalb eng zusammenarbeiten und wenn möglich gemeinsame Versuchsprogramme ausarbeiten.

Ein weiterer Forschungsschwerpunkt könnte der Themenkreis Kathodenmaterialien mit hoher regenerierbarer Ladungsdichte $(\mathrm{Ah} / \mathrm{kg})$ sein. Von besonderem Interesse sind Interkalationselektroden oder Insertionselektroden (z. B. $\mathrm{V}_{6} \mathrm{O}_{13}, \mathrm{MnO}_{2}$, $\mathrm{TiS}_{2}$ usw.). Die Erfassung der Diffusionsund Migrationsprozesse sowie die Aufklärung der Kristallstruktur und deren Modifikation bei der Einlagerung der Insertions- oder Interkalationsionen ist hier wichtig für die Selektion von geeigneten Elektrodenmaterialien. Auch thermochemische Untersuchungen dieser Prozesse sind nötig, wenn wir vom gewöhnlichen 'Herumpröbeln' wegkommen wollen.

Dieser Themenkreis hat zudem eine sehr grosse Überlappung mit dem zuerst erwähnten Themenkreis 'Leitende Polymere' und 'Redoxpolymere'.

Neben diesen Themenkreisen, die vor allem für die Entwicklung von neuen wiederaufladbaren Batterien mit hoher Energiedichte wichtig sind, gibt es aber auch sinnvolle Forschungsschwerpunkte, die zur Entwicklung von effizienten Brennstoffzellen oder von Metall/Luft-Batterien von Interesse sind. Dazu gehört: Entwicklung von Gas-Elektroden (z. B. $\mathrm{H}_{2}-, \mathrm{O}_{2}$-Elektroden), Reduktion von $\mathrm{CO}_{2}$ und $\mathrm{N}_{2}$ zur Herstellung energiereicher Produkte mit Hilfe der Elektrokatalyse. Hier müssen Stofftransporte an Dreiphasengrenzen und heterogene Reaktionsmechanismen zum Verständnis der Katalyseprozesse studiert werden. Daneben sind grosse Anstrengungen hinsichtlich der Makro- und Mikrostruktur der Elektroden bis hin zum molekularen Niveau nötig.

Dieses Gebiet hat eine sehr starke Überlappung mit wichtigen Themen des Programms Photochemie (z.B. photochemische Reduktion von $\mathrm{CO}_{2}$ oder $\mathrm{H}_{2} \mathrm{O}$ ). Eine enge Zusammenarbeit über die Programmgrenzen der Elektrochemie hinaus ist hier sicher erstrebenswert.

Für dic Anwendung in Brennstoffzellen wird es wichtig sein, dass die Gas-Elektroden grosse Stromdichten erreichen und dass mit einer äusserst kompakten Bauweise die bis heute erreichten Leistungsdichten wesentlich verbessert werden können. Gute Aussichten haben dabei Kompositelektroden. Bei diesen Elektroden wird der Katalysator in hochdisperser Form in ein leitendes Polymer mit guter Ionenleitfähigkeit und hoher Gasdurchlässigkeit eingebettet und auf einen elektrisch leitenden, porösen Träger aufgezogen.

Wichtige Forschungsthemen, die den Forschungsschwerpunkten übergeordnet sind, sind die Materialtechnologie, die Entwicklung neuer Insertions- und Interkalationselektroden, die Methodenentwicklung zur Verfolgung der in den Zellen ablaufenden Teilprozesse und die Modellierung von Elektrodenprozessen und Zellsystemen.

\section{Ausblick 'Elektrochemische Energiespeicherung'}

Die vorgeschlagenen, recht aufwendigen Forschungsarbeiten liessen sich schwer rechtfertigen, wenn nicht zumindest theoretisch gezeigt werden könnte, dass umweltfreundliche, wiederaufladbare Batterien mit grosser Energie- und Leistungsdichte grundsätzlich möglich sind.

Es würde zu weit führen, hier alle möglichen Neuentwicklungen auf dem Gebiet der Sekundärbatterien aufzuzählen. Einige grundsätzliche Überlegungen an ausgewählten Beispielen sollen zur Illustration der Problematik kurz erwähnt werden.

Die Batterien bestehen im Prinzip nur aus drei funktionswichtigen Bestandteilen. Es braucht eine Anode, eine Kathode und einen ionenleitenden Elektrolyten. Dabei gibt es im wesentlichen zwei Typen von wiederaufladbaren Batterien. Der erste Typ funktioniert ähnlich wie z. B. der BleiAkkumulator. Beim Lade- oder beim Entladeprozess werden die an den Elektroden gebildeten Produkte im Elektrodenzwischenraum im Elektrolyten deponiert. So wird z. B. beim Ladeprozess des Blei-Akkumulators $\mathrm{H}_{2} \mathrm{SO}_{4}$ gebildet oder beim Entladeprozess der $\mathrm{Zn} / \mathrm{Br}$-Batterie $\mathrm{ZnBr}$, in den Elektrolyten abgegeben. Die Zelle muss so gebaut sein, dass die Produkte im Elektrolytenvolumen gespeichert werden können.

Der zweite Typ verwendet Interkalations- oder Insertionselektroden. Bei diesen Zellen werden Ionen an einer Elektrode freigesetzt und von der andern Elektrode wieder eingebaut. Das ElektrolytVolumen kann sich dabei auf ein Minimum beschränken, weil hier beim Lade-

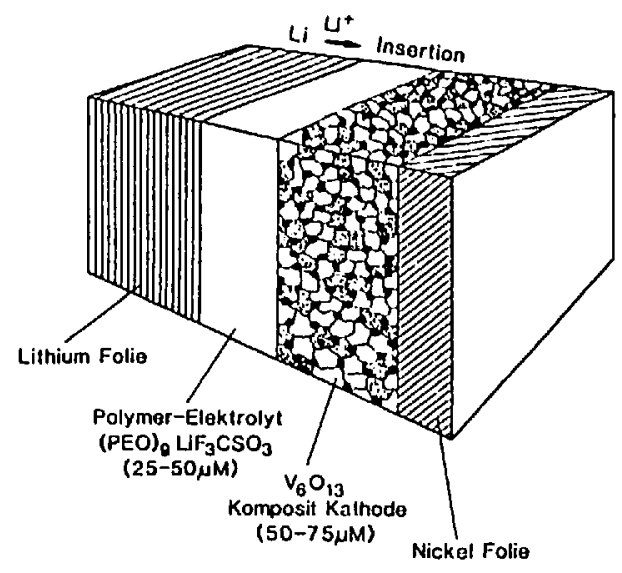

- Kohle

$\odot v_{0} O_{1}$,

O Polymer

Fig. 1. Aufbau einer $\left(\mathrm{Li} / \mathrm{V}_{\mathrm{b}} \mathrm{O}_{13}\right)$-Dünnschicht-Batterie 


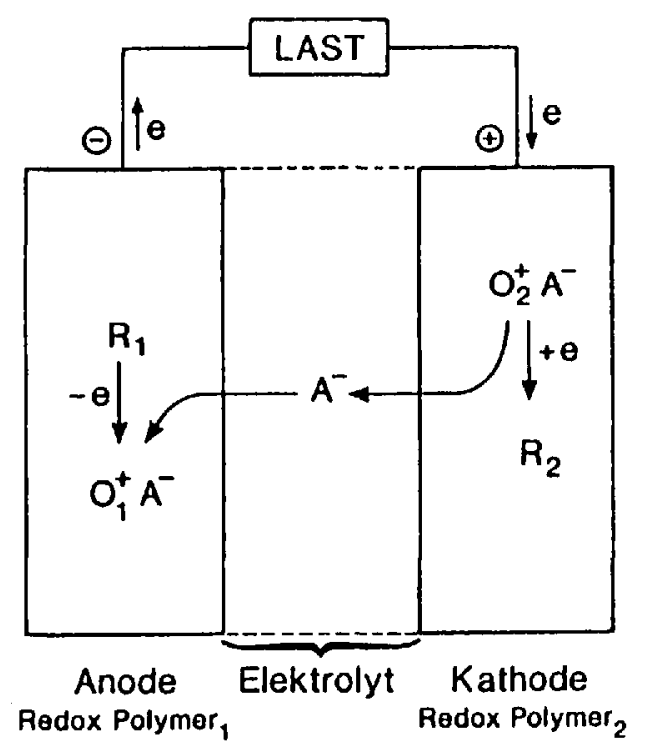

$$
E_{R_{1} / O_{1}}^{O}<E_{R_{2} / O_{2}}^{O}
$$

Fig. 2. Prinzip einer (Redox-Polymer)Batterie mit Anionen-Transfer

und Entladeprozess keine Produkte im Elektrolyten deponiert werden müssen. Mit diesen Zellen können prinzipiell höhere Energiedichten erreicht werden. Die dabei herumgeschobenen Ionen können sowohl Kationen als auch Anionen sein. Als Beispiel einer Batterie mit einer Kationen einlagernden Elektrode ist in Fig. 1 der Aufbau einer ( $\mathrm{Li} / \mathrm{V}_{6} \mathrm{O}_{13}$ )-Dünnschichtbatterie aufgezeichnet [1]. In Fig. 2 wird das Prinzip einer Redox-Polymer-Batterie mit Anionen-Transfer dargestellt. Ein besonders lohnendes Forschungsziel ist die Entwicklung von neuen Insertions- oder Interkalationselektroden mit möglichst wenig Molmasse pro einlagerbarem Ion. Damit die theoretische Energiedichte der in Fig. 2 abgebildeten Zelle diejenige des Blei-Akkumulators übersteigt, müsste die Redoxpotentialdifferenz der beiden Polymeren mehr als $1,5 \mathrm{~V}$ betragen und die totale Molmasse der Polymeren pro verschobenes Ion und Elektron (inklusive des verschiebbaren Ions) kleiner als $240 \mathrm{~g}$ sein. Dies ist durchaus möglich. Es ist auch nicht auszuschliessen, dass Redoxpolymere mit grösseren Potentialdifferenzen und wesentlich kleineren Molmassen gefunden werden können. Die untere Grenze für die Summe der Molmassen der beiden Elektroden liegt bei $c a .100 \mathrm{~g}$. Dies bedeutet, dass diese Batterien prinzipiell wesentlich mehr als doppelt so grosse Energiedichten als der Blei-Akkumulator erreichen könnten. Falls es gelingt, eine der beiden Polymer-Elektroden mit einer Al-, $\mathrm{Mg}$ - oder evtl. Na-, K-Elektrode zu ersetzen und falls Polymere gefunden werden, die die Ionen dieser Elektrodenmaterialien einlagern können, dann sollten noch wesentlich höhere Energiedichten erreichbar. sein. Dies vor allem deswegen, weil die Molmasse pro austauschbares Elektron bei diesen Elektroden sehr klein ist (beim Al z. B. nur 9 g/e). Praktische Energiedich- ten von $200 \mathrm{Wh} / \mathrm{kg}$ sollten auf lange Sicht erreichbar sein (Systeme mit theoretischen Energiedichten von $800-1000 \mathrm{Wh} / \mathrm{kg}$ sind von der Chemie her prinzipiell möglich, praktisch aber äusserst schwer zu verwirklichen).

Die wiederaufladbaren Batterien (Sekundärbatterien) werden mit einigen Ausnahmen für spezielle Anwendungen noch immer von den Blei-Akkumulatoren dominiert. Obwohl diese Batterie bereits mehr als 100 Jahre alt ist, wird wahrscheinlich auch heute noch am meisten Geld für die Verbesserung des Blei-Akkus eingesetzt. Hinsichtlich Energiedichteverbesserung sind die Möglichkeiten jedoch relativ beschränkt. Die meisten Blei-Akkumulatoren haben eine Energiedichte von 20-40 Wh/kg. Die Energiedichte kann wahrscheinlich maximal auf $c a .60 \mathrm{Wh} / \mathrm{kg}$ erhöht werden. Die theoretische Energiedichte (nur aktives Elektrodenmaterial + stöchiometrische Menge $100 \%$ $\mathrm{H}_{2} \mathrm{SO}_{4}$ ) beträgt $167 \mathrm{Wh} / \mathrm{kg}$. In der Regel kann in praktischen Zellen ca. $25-30 \%$ des theoretischen Wertes erreicht werden.

Es gibt heute einige recht weitentwikkelte Sekundär-Batterien, die in naher $\mathrm{Zu}$ kunft den Blei-Akkumulator konkurrenzieren können, so z. B. $\mathrm{Zn} / \mathrm{Br}_{2}$-Batterie oder evtl. auch die ( $\mathrm{Zn} / \mathrm{NiOOH})$-Batterie und $(\mathrm{Fe} / \mathrm{NiOOH})$-Batterie. Diese Batterien haben eine Energiedichte, die um 50$100 \%$ höher liegt als beim Blei-Akkumulator. Noch etwas besser sind die recht weit entwickelten, aber zu teuren $\left(\mathrm{Na} / \mathrm{S}_{\mathrm{x}}\right)$ - und $\left(\mathrm{Ni} / \mathrm{H}_{2}\right)$-Batterien. Letztere hat sich vor allem in der Raumfahrt wegen ihrer grossen Zyklenzahl bewährt.

In letzter Zeit ist es auch gelungen, wiederaufladbare Li-Batterien herzustellen. Die Energiedichte dieser Batterien ist besonders gross (bis $120 \mathrm{Wh} / \mathrm{kg}$ ), doch sind sie nicht ganz ungefährlich. Interessant wäre, wenn die Li-Elektrode in dieser Batterie gegen eine weniger gefährliche $\mathrm{Mg}$-, Al- oder Ca-Elektrode ausgetauscht werden könnte. Sehr hohe Stromdichten von mehreren $\mathrm{A} / \mathrm{cm}^{2}$ erhält man mit ( $\mathrm{LiAl} /$ $\mathrm{FeS}_{2}$ )-Zellen in Schmelzen. Die hohen Betriebstemperaturen schränken die Anwendungsmöglichkeiten dieser Zellen jedoch stark ein. Dies ist auch bei den $\left(\mathrm{Na} / \mathrm{S}_{\mathrm{x}}\right)$ Batterien der Fall.

Bei den Brennstoffzellen haben wir eine ähnliche Situation wie bei den Photozellen. Ihre Nützlichkeit und Funktionstüchtigkeit konnte bei Raumfahrtprojekten und in Demonstrationsanlagen mit Leistungen bis hin zu mehreren Megawatt eindrücklich demonstriert werden. Die ersten Methanol-Brennstoffzellen werden offenbar für gewisse Anwendungen bereits kommerzialisiert. Für das Elektromobil und die dezentrale Stromproduktion sind die Kosten pro Leistungseinheit jedoch noch zu hoch. Billiger werden die BrennstoffZellen nur, wenn sie auch in grossen Massen fabriziert werden können.

Kompakte Zellen mit sehr hoher Stromdichte können vor allem mit protonenleitenden Membranen gebaut werden. Bei diesen Zellen mit sauren Polyelektrolyten werden Katalysator und Leiterplatten beidseitig direkt auf die sehr dünne Membran aufgebracht. Leider sind diese Membranen (Nafion- oder Dow-Membrane) sehr teuer. Dazu kommt, dass in sauren Elektrolyten bis jetzt praktisch nur Edelmetalle als Katalysatoren verwendet werden konnten. In alkalischen Elektrolyten sind auch billigere Katalysatoren möglich, so z.B. Silberoxid oder Aktivkohle, die vorgängig mit stickstoffhaltigen Verbindungen pyrolysiert wurden. Ein sehr lohnendes Forschungsthema wäre die Entwicklung einer billigen $\mathrm{H}^{+}$-ionenleitenden oder einer $\mathrm{OH}^{-}$-ionenleitenden Membran, wobei auch anorganische, kunststoffgebundene Membranen denkbar sind.

Die Metall/Luft-Zellen haben eine ausserordentlich hohe Energiedichte. Diese ergibt sich vor allem deshalb, weil im Unterschied zu den sekundären Batterien einer der Reaktionspartner (Luft) nicht gespeichert werden muss und weil im Unterschied zu den $\left(\mathrm{H}_{2} / \mathrm{O}_{2}\right)$-Brennstoff-Zellen der zweite Reaktionspartner in sehr kompakter Form (fest statt gasförmig) vorliegt. Dazu kommt, dass recht viele Metalle hohe Oxidationsenthalpien haben. Ein grosser Teil des DOE (Department of Energy)-Budgets in den USA wird in die Erforschung dieser Zellen investiert. Ihr Entwicklungsstand ist jedoch im Moment noch viel weniger weit als derjenige der Brennstoffzellen. Wichtig für die Weiterentwicklung dieser Zellen ist die Abklärung des Reaktionsmechanismus an diesen meist mit einer Oxid-Schicht überzogenen Metall-Elektroden. Detaillierte Kenntnisse über den Elektronen- und Ionentransport in der Oxid-Schicht sowie deren Beeinflussungsmöglichkeiten sind von ausschlaggebender Bedeutung für eine gezielte Weiterentwicklung dieser potentiell interessanten Zellen. Ein weiteres Problem ist die kontinuierliche Abtrennung der Reaktionsprodukte aus dem Elektrolyten und deren Entsorgung resp. Rückführung zu Metall.

\section{Forschungsplan}

Es soll zuerst in einer zwei- bis dreijährigen, grundlagenforschungsorientierten Phase das Gebiet der elektrochemischen Energiespeicherung möglichst breit bearbeitet werden. Dabei ist es sehr wichtig. dass die einzelnen Forschergruppen untereinander einen guten Kontakt haben. Dies soll mit gemeinsamen Seminaren und Symposien gefördert werden.

Damit die einzelnen Forschungsteams nicht nur zusammenhanglose Beiträge auf dem weiten Gebiet der elektrochemischen Energiespeicherung leisten, sollen von Anfang an einige Fernziele anvisiert werden. Zur Verfolgung dieser Ziele können dann laufend erstrebenswerte Meilensteine definiert werden. Nach zwei bis drei Jahren sollen die Ziele dann weitgehend konkretisiert sein. 
Aus heutiger Sicht kann folgendes gesagt werden: Erste Priorität haben die Projekte, die im Zusammenhang mit wiederaufladbaren, energiedichten Batterien und Brennstoff-Zellen mit grosser Leistungsdichte stehen. Diese Batterien und Brennstoff-Zellen haben das breiteste Anwendungsspektrum. Die Förderung dieser Forschungsarbeiten ist deshalb auch be sonders lohnend. Vorhanden sind zur Zeit vor allem Resultate und Erfahrungen aus der Forschung an (Al/Redox-Polymer)Batterien. Die Erforschung von Al-Batterien wird am PSI seit einigen Jahren durch den Nationalen Energieforschungsfonds (NEFF) gefördert. Diese Resultate sind auch im Zusammenhang mit der Entwicklung von anderen (M/Redox)-PolymerZellen (M: Li, K, Na, Ca, Mg, Zn) oder Redox-Polymer $/ \mathrm{MCl}_{x}$ resp. Redox-Poly$\operatorname{mer} / \mathrm{MO}_{\mathrm{x}}(\mathrm{M}: \mathrm{Fe}, \mathrm{Ni}, \mathrm{Co}, \mathrm{V}, \mathrm{Cu})$ von Interesse.

Speziell für das Elektroauto sind auch die Zellen mit $\mathrm{O}_{2}$-Elektroden interessant Im Prinzip sind mit diesen Stromquellen die energiedichtesten Batterien möglich. Die Entwicklung dieser Zellen ist aber sehr aufwendig. In einer späteren Phase kann es sinnvoll sein, wenn sich die Forschergruppen auf das aussichtsreichste Zellsystem konzentrieren.

Die elektrochemische Produktion energiereicher Produkte ist langfristig wichtig, in unmittelbarer Zukunft jedoch kaum von volkswirtschaftlichem Interesse. Diese Aussage stimmt, solange der Preis für elektrische Energie im Vergleich zum Preis der in fossilen Brennstoffen gespeicherten Energie hoch ist. Forschung auf dem Gebiet der Elektrokatalyse ist im Zusammenhang mit der elektrochemischen Oxidation von $\mathrm{MeOH}$ und der Reduktion von $\mathrm{O}_{2}$ förderungswürdig. Die Reduktion von $\mathrm{CO}_{2}$ und $\mathrm{N}_{2}$ hat jedoch zweite Priorität.

\section{Eingegangene Projekte}

\subsection{Allgemeine Bemerkungen}

Die Reaktionen auf die Ausschreibung der Forschungsprogramme 'Chemische Energiespeicherung' haben gezeigt, dass das Programm 'Elektrochemie' weitaus das grösste Echo gehabt hat. Dies, obwohl im Schulratsbereich (Ausnahme PSI) die Elektrochemie weitgehend vernachlässigt wird. Das recht weitgestreute Interesse am Programm 'Elektrochemie' hängt wahrscheinlich damit zusammen, dass elektrochemische Energiespeicher-Systeme am ehesten zu Produkten führen, die vermarktet werden können. Zudem stellt Elektrizität eine äusserst hochwertige Energieform dar. Wer fragt schon danach, wie teuer die in einer Taschenlampenbatterie gespeicherte $\mathrm{kWh}$ ist? Ganz anders ist dies bei der Speicherung thermischer Energie oder beim $\mathrm{H}_{2}$. Hier steht die gespeicherte Energie immer in Konkurrenz mit fossilen Brennstoffen. Ein weiterer Grund für das grosse Interesse am Programm 'Elektrochemie' dürfte darin zu finden sein, dass im
Programm neue Materialien entwickelt werden sollen, die auch in vielen anderen Gebieten zur Anwendung kommen können. Deshalb setzt auch das DOE in den USA wesentlich mehr Geld für die elektrochemische Energiespeicherung ein als für $\mathrm{H}_{2}$ und Thermochemie zusammen.

Mit den in Angriff genommenen Projekten können für die Schwerpunktsthemen 'Leitende Polymere, Redox-Polymere, Membranen, Polyelektrolyte' und 'Untersuchung von Kathodenmaterialien mit hoher Energiedichte und Herstellung energiereicher Produkte' sowie für die 'Entwicklung von Gas-Elektroden, Reduktion von $\mathrm{O}_{2}, \mathrm{CO}_{2}$ und $\mathrm{N}_{2}$ Elektrokatalyse interessante Forschungsteams zusammengestellt werden. In diesen Forscherteams sind die Materialwissenschaftler aus den Disziplinen Festkörperphysik und Festkörperchemie noch zu wenig vertreten. Hingegen ergibt sich erfreulicherweise eine recht starke Gruppe mit Interesse auf dem Gebiet der numerischen Simulation von Elektrodenprozessen und Modellierung von ganzen Zellsystemen. Zum Thema Methodenentwicklung (in-situ-Analyse-Methoden zur Untersuchung der Elektrodenprozesse) kann auch eine leistungsfähige Gruppe gebildet werden. In einem schon früher vom BEW unterstützten Projekt wird geprüft, ob sehr dünne Pd/Ag-Membranen für die Herstellung einer (MeOH/Luft)-Brennstoffzelle verwendet werden können.

\subsection{Kurzbeschreibung der einzelnen Forschungsprojekte}

\section{Elektrolytische $\mathrm{CO}_{2}$-Reduktion}

$\mathrm{CO}_{2}$ soll mit Hilfe von festen Alkali-Hydroxiden oder Alkali-Carbonaten oder deren Lösungen in $\mathrm{H}_{2} \mathrm{O}$ aus Luft absorbiert werden. Die dabei gebildeten Carbonate oder Hydrogencarbonate werden anschliessend als Schmelze oder in Lösung einer Elektrolyse unterworfen. Durch geeignete Elektrolyse-Bedingungen will man versuchen, $\mathrm{CO}_{2}$ zu $\mathrm{MeOH}$ oder EtOH zu reduzieren. Das Ziel ist, $\mathrm{CO}_{2}$ als Bestandteil eines flüssigen Energieträgers im Kreislauf zu führen. Seine Langzeitspeicherung fälit weg. Es wird mit den Luftströmungen in der Atmosphäre transportiert.

Kontaktadresse: Dr. H. Grüninger, TCL, ETH-Zentrum, 8092 Zürich, Tel. 01/ 2563585

\section{Alkalische (MeOH/Luft)-Brennstoff-Zelle}

Es sollen die Grundlagen für die Realisierung einer mobilen, alkalischen (MeOH/Luft)-Brennstoff-Zelle mit vorgelagertem 'Reforming' von $\mathrm{MeOH}$ erhoben werden. Das dabei anfallende, für den alkalischen Elektrolyten schädliche $\mathrm{CO}_{2}$ soll mittels einer (Pd/Ag)-Membran abgetrennt werden, welche gleichzeitig als Anode dient. Die primären Hauptziele des Projekts sind einerseits die Entwicklung einer trägergestützten ( $\mathrm{Pd} / \mathrm{Ag})-\mathrm{Membran}$ und andererseits die Erprobung von Reformierkatalysatoren bzw. die Dimensionierung des Katalysator-Betts. Ausserdem sollen die in der vorangegangenen Projektphase begonnenen Arbeiten über organische Sauerstoff-Katalysatoren abgeschlossen werden.

Kontaktadresse: Prof. W. Richarz und Th. Allmendinger, Technisch-Chemisches Laboratorium, ETH-Zentrum, 8092 Zürich. Tel. 01/2563091

'Numerical Description of Non-stationary Processes at Polymer-coated Electrodes'

La description et l'interprétation numérique de processus non-stationaires d'électrodes recouvertes d'un film de polymère redox sont vitales pour une bonne compréhension de résultats expérimentaux. Dans ce but, une méthode de calcul numérique sur ordinateur est développée permettant le traitement de presque toutes les techniques de mesures électrochimiques telles que: polarographie, voltamétrie cyclique, chronoampérométrie, mesure d'impédance en courant alternatif, déflection de faisceau laser, etc. Des études préliminaires ont montré que la méthode que nous avons mise au point, basée sur les éléments finis et l'intégration semi-implicite, permet de réaliser le but assigné.

Kontaktadresse: PD Dr. C. Daul, Institut für anorganische Chemie, Universität Fribourg, Pérolles, 1700 Fribourg, Tel. 037/ 826436

Modellierung von elektrochemischen Systemen, angewandt auf die Abscheidung und Auflösung von Metallen

Das Projekt wurde am 1.Januar 1990 gestartet. Projektziel ist die Entwicklung quantitativer Methoden zur Simulation des Verhaltens von elektrochemischen Systemen mit mehreren Reaktionen oder mit Form-Änderungen der Elektroden sowie die experimentelle Untersuchung solcher Systeme einschliesslich der Entwicklung von dafür geeigneten Zell-Anordnungen. Als Simulationsmethode für Probleme der Stromdichteverteilung steht die 'boundary element method' (BEM) im Vordergrund, da sie sich besonders gut eignet, um Formänderungen und nicht lineare Randbedingungen zu behandeln. Ein Teil des Forschungsprojektes wird in Form einer Doktorarbeit ausgeführt werden.

Kontaktadresse: Prof. Dr. D. Landolt, EPFL, DMX, 34, chemin de Bellerive, 1007 Lausanne, Tel. $021 / 6932981$

Beeinflussung des Redox-Potentials von Aluminium in Raumtemperatur-Schmelzen

Optimierung von RaumtemperaturSchmelzen, insbesondere von Trimethyl(phenyl)ammonium-chlorid/AICl ${ }_{3}$, als Elektrolyt zur verlustarmen, reversiblen Abscheidung und Auflösung von $\mathrm{Al}$ im Hinblick auf die Verwendung von $\mathrm{Al}$ als Energiespeicher.

Insbesondere ist der Einfluss der Verdünnung der Schmelze mit inerten Lösungsmitteln, der Zusatz von Komplexbildnern und von Metall-Halogeniden im Hinblick auf die Optimierung des Elektrolyten zu untersuchen. 
Kontaktadresse: Prof. F.E. Emmenegger, Inst. für anorganische Chemie, Universität, Pérolles, 1700 Fribourg, Tel. 037/ 826422

Elektrophile, monomere und dimere Metalloporphyrine als Oberflächen-Katalysatoren

Systematische Entwicklung elektrophiler, porphinoider Metall-Komplexe als elektrochemisch einsetzbare, oberflächengebundene Katalysatoren für die Reduk. tion von $\mathrm{O}_{2}$ zu $\mathrm{H}_{2} \mathrm{O}$ (Problematik: Brennstoff-Zellen).

Kontaktadresse: PD Dr. B. Kräutler, Laboratorium für Organische Chemie der ETH, Universitätstr. 16, 8092 Zürich, Tel. 01/2564513

'Fixation de l'azote moléculaire par réduction électrochimique'

Notre projet consiste en une étude du concept d'une électrode (cathode) permettant d'intégrer simultanément le réducteur de l'azote, le catalyseur ainsi que l'inhibiteur de la réaction secondaire (réduction de l'eau). Parmi les réducteurs chimiques de l'azote les plus efficaces se trouvent les hydroxydes de certains métaux de transition ( $\mathrm{V} / \mathrm{Ti}, \mathrm{Cr}$ ) contenant un composé de molybdène comme catalyseur. Les électrodes en oxydes, étudiées depuis plusieurs années dans notre laboratoire, pourraient former de telles structures permettant de réaliser un contact direct entre l'oxyde réducteur et le catalyseur (immobilisé). Ces électrodes constituent également un support adéquat pour les complexes catalysant la fixation de l'azote.

Kontaktadresse: Dr. Jan Augustynski, Chimie appliquée, Sciences II, 30, quai E. Ansermet, 1211 Genève 4, Tel. 022/ 7026413

\section{'Conductive Polymers for Rechargeable Batteries'}

Organic polymers can be used as electrodes in rechargeable batteries. In combination with electroactive metal anodes, such as $\mathrm{Li}, \mathrm{Na}$ or $\mathrm{Al}$, with organic or molten-salt anhydrous electrolytes, there is the potential for high energy densities in these batteries. Among the conductive polymers being investigated is polyaniline and also polymethylthiophene.

Kontaktadresse: Prof. Dr. M. Grätzel, Inst. de chimie physique 2, EPFL, 1015 Lausanne, Tel. 021/6933689

\section{Polymere als Lösungsmittel in elektroche- mischen Zellen}

Solvatation von Ionen in Polymeren Wechselwirkung Elektrolyt-Polymer, Komplexierung von Metallionen, Dynamik von Metallionen.

Kontaktadresse: Prof. C.W. Schläpfer, Inst. für anorg. und analytische Chemie, Universität Fribourg, Pérolles, 1700 Fribourg, Tel. 037/826430/22
'Modélisation d'un réacteur électrochimique et vérification expérimentale'

Elaboration d'un logiciel permettant de simuler le fonctionnement d'un réacteur électrochimique bipolaire et vérification expérimentale.

Kontaktadresse: Hubert Froidevaux, DMA, EPFL, 1015 Lausanne, Tel. 021/ 6932544

Elektrochemische Impedanz-Spektroskopie an stromleitenden und Redox-Polymeren

Die Aufklärung der Kinetik ausgewählter Polymer-Elektroden in der Absicht, verallgemeinerbare theoretische und empirische Unterlagen zur Evaluation und gegebenenfalls Optimierung derartiger Systeme als elektrochemische Energiespeicher bereitzustellen.

Kontaktadresse: Prof. E. Schmidt, Inst. für anorganische, analytische und physikalische Chemie, Freiestr. 3, 3003 Bern 9, Tel. $031 / 654216$

'Préparation et caractérisation de catalyseurs dispersés dans des couches des polymères conducteurs'

Préparation des couches des polymères conducteurs (polyaniline et polypyrrole) en couche mince contenant des microparticules métalliques $(\mathrm{Pt})$ disperées; étude de leurs propriétés électrocatalytiques: I) réactions électrochimiques simples; 2) réactions électrochimiques plus complexes (p. ex.: l'oxydation de l'acide formique, du méthanol et du glucose).

Kontaktadresse: $M$. Koudelka, IMT, Brequet 2, 2000 Neuchâtel, Tel. 038/246000

\section{Polymer-Anode für (Polymer-Polymer).} Batterie

1) Optimierte Herstellung von Polypyrrol als Anodenmaterial. Auswahl geeigneter Substrate (die das Material halten, den Strom abführen und auch bei der elektrochemischen Herstellung als Elektrode dienen) und Elektrolyte. Charakterisierung des Anodenmaterials mit physikalischen Methoden.

2) Grösstmögliche Verminderung der Selbstentladung von Polypyrrol-Anoden (wie auch der Kathoden und der ganzen Batterie).

3) Aufbau einer Prototyp-Zelle mit Kathode und Anode aus Polypyrrol (die Kathode ist bereits bekannt), Charakterisierung der Eigenschaften als Grundlage für eine eventuelle industrielle Weiterentwicklung.

Kontaktadresse: Dr. Klaus Müller, 7 , route de Drize, 1227 Carouge, Tel. 022/ 270270 oder $022 / 270302$

Neuartige Membranen für saure Brennstoff-Zellen und Wasserelektrolyseure

Untersuchung des Degradationsmechanismus der Polymer-Membranen in $\mathrm{CH}_{2}$ $\mathrm{O}_{2}$ )-Membranzellen, Einfluss der Betriebsparameter und der Elektrodenmaterialien. Synthese von kostengünstigen Membra- nen mit optimierten Eigenschaften (Leitfïhigkeit, Elektroosmose, Stabilität).

Kontaktadresse: Dr. Günther G. Scherer, PSI, 5232 Villigen, PSI, Tel. 056/992111

Simultan-Untersuchungen der optischen Absorption und Voltammogramme von leitenden Polymeren und Redox-Polymeren

Durch optische und elektrochemische Messungen sollen die Redox-Vorgänge in leitenden Polymeren und Redox-Polymeren charakterisiert werden.

Kontaktadresse: H. Kiess, PSI c/o Lab. RCA Ltd., Badenerstr. 569, 8048 Zürich, Tel. 01/4926350

Untersuchung der elektrochemischen Auflösung und Abscheidung von $\mathrm{Mg}$ und $\mathrm{Al}$ in nichtwässrigen Lösungsmitteln

Energiespeicherung mit $\mathrm{Al}$ und $\mathrm{Mg}$. In organischen Elektrolytsystemen soll die Elektrochemie von Al und $\mathrm{Mg}$ untersucht werden. Dabei sollen die Reaktionskinetik und die Massentransport-Phänomene be der elektrochemischen Auflösung und $\mathrm{Ab}$ scheidung dieser Metalle untersucht werden.

Kontaktadresse: Dr. Helmut Tannenherger, CSEM SA, Rue A.-L. Breguet 2, 2000 Neuchâtel 7, Tel. 038/240161

In-situ-Rastertunnelmikroskopie an nichtmetallischen Elektrodensubstraten

In Zusammenarbeit mit andern Arbeitsgruppen des Programms Elektrochemie sollen elektrolytische Rastertunnelmikroskopie-Untersuchungen an verschiedenen Elektrodensystemen durchgeführt werden. Mit dieser neuen In-situ-Technik sollen speziell an leitenden Polymeren und $\mathrm{Re}$ dox-Polymeren Informationen über die Oberflächenstruktur, den Ladungsdurchtritt und die Elektrodenkinetik erarbeitet werden.

Kontaktadresse: PD Dr. H. Siegenthaler. Inst. f. anorganische, analytische und physikalische Chemie, Universität Bern, Freiestr. 3, 3000 Bern, Tel. 031/654219

Den Herren Dr. G. Schriber, BEW, und Dr. P.E. Zinsli, BBW, Dr. F. Casal, CORE, Dr. P. Kessefring, PSI, sei hier für ihre tatkräftige Unterstützung des Projektes 'Elektrochemie' herzlich gedankt. Ein spezieller Dank geht an Dr. E. Deiss und Frau U. Grütter für ihre administrative Hilfe.

Bemerkung: Der Autor möchte darauf hinweisen dass 1991 vom 25. bis 30. August in Montreux cine Konferenz der "International Socicty of Electrochemistry' stattfindet. An dieser Konferenz werden wichtige Aspekte der elektrochemischen Energicspeicherung und -umwandlung ausführlich diskutiert.

Eingegangen am 7. März 1990

[1] A. Hooper, Chem. Ind. 1986, 198201 